\title{
Coupled system of PDEs to predict the sensitivity of some materials constituents of FOUP with the AMCs cross-contamination
}

\author{
N. Santatriniaina ${ }^{1}$, J. Deseure ${ }^{2}$, T.Q. Nguyen $^{3}$, H. Fontaine ${ }^{3}$, C. Beitia ${ }^{3}$, L. Rakotomanana ${ }^{1}$ \\ ${ }^{1}$ University of Rennes, Mathematical Research Institute of Rennes, 263 av. du Général Leclerc, 35042 Rennes, France \\ ${ }^{2}$ University of Joseph Fourier, PHELMA Campus, 1130 rue de la piscine, 38402 Grenoble, France \\ ${ }^{3}$ Atomic Energy Commission, Laboratoire d'Electronique et Technologies d'Information, LETI/DTSI, 17 rue des Martyrs, \\ 38054, Grenoble Cedex 9, France \\ *Corresponding author E-mail: nirina.santatriniaina@univ-rennes1.fr
}

Copyright $\odot 2014$ N. Santatriniaina et al. This is an open access article distributed under the Creative Commons Attribution License, which permits unrestricted use, distribution, and reproduction in any medium, provided the original work is properly cited.

\begin{abstract}
This paper deals a predictive model using modeling and computational methods to investigate the sensitivity of some materials constituents of the FOUP with the AMCs cross contamination. Required numerical tools which are employed in order to study the AMCs cross-contamination transfer phenomena between wafers and FOUPs were developed. Numerical optimization and finite elements formulation in transient analysis were established. Analytical solution of one dimensional problem was developed and the calibration process of physical constants was performed. This mode was used to study the sensitivity of some material with the cross contamination. The behavior of the AMCs in transient analysis was determined. The model framework preserves the classical forms of the diffusion and convection-diffusion equations and yields to consistent form of the Fick's law. The adsorption kinetics of the contaminant on the surface (interface contaminant/polymer) was assumed. The adsorption process and the surface roughness effect were also traduced as a boundary condition using the switch condition Dirichlet to Neumann and the interface condition. Many tests of contamination processes were assumed in order to study the sensitivity of the materials. Optimization methods with analytical solution were used to define physical constants for each material versus contaminant. Finite element methods including adsorption kinetic were also used and by using Henry law on the interface and the switch of Dirichlet to Neumann conditions.
\end{abstract}

Keywords: Cross-contamination, FOUP, mathematical modeling, finite element method, sensitivity study.

\section{Introduction}

In high-tech microelectronics engineering, more attention is required to challenge the contamination control during the manufacture of integrated circuit (I.C). Integrated circuits are manufactured from a monocristallin silicium plates (wafer) [1]. Minutiarization of the I.C is designed with the 45, 32, 22 [nm], in fact, the wafer's surface is very sensitive to molecular contaminations of the wafer are critical subjects which can potentially cause defect on the use and have impacts in the device performance [2], [3]. In this work, we keep more attention in molecular contamination that can damage and induce a significant impact in manufacturing yields. The wafer carrier and storage plays a significant role for contamination control [4]. The use of the Front Unified Pods (FOUPs) to transport from tool to tool 25 wafers in the $300[\mathrm{~mm}]$ are necessary to protect the wafer against contamination mainly the Airborne Molecular Contamination. This container may contain a lot of contaminant including, there so called AMCs or Airborne Molecular Contaminants and can still contain a significant amount of contamination with the potential to damage the wafer. This enclosed minienvironment is made of porous polymers, mainly in PC, COP, PP, PEEK and PEI [5], [6].

These materials are known with their adsorption and outgas properties. They are also able to absorb volatile compounds present in the atmosphere coming from the connection to equipment or from the fresh assembly of wafers just processed (post processed wafers). During the storage, these wafers may outgas the chemicals used with during the process. As 
results, a reversible and an irreversible outgassing of contaminant previously trapped in polymer is possible. In fact a contemned FOUP already itself may be a source of contamination because it already adsorbs the contaminant from the wafers. This cross-contamination scheme was clearly evidenced for volatile acids in presence. Many works have been published which deal with experiment measurement method to quantify and to investigate this phenomena for each types of material.

Generally, when one object becomes contaminated by either direct or indirect contact with another object which is already contaminated, we talk about cross contamination. In microelectronics industry, this process generally takes place at FOUPs which contain the wafers before and after production. The main object sources of the contamination in microelectronic factory are the wafer, air, FOUP by which a new wafer may be contamined before the manufacturing processes.

Some manufacture processes such as dry engraving (plasma), depot and photolithography are the source of contamination, when the wafer is already processed its surface is contamined by the volatile acids [5], [6]. After this manufacturing process, these wafers will be stored in the FOUP. Then, the wafer is exposed to the FOUPs atmosphere and an acid pollution may happen from wafer to FOUP by the intermediate air (atmosphere). As outlined before, the FOUP material can absorb the contaminant in it's around, a adsorption phenomenon from air to the internal surface of the FOUP followed by diffusion in volume happens. When the wafer moved or the FOUP is opened, the air in the FOUP atmosphere changes and desorption phenomena takes place, a cross contamination from FOUP to new wafer may happen i.e. contamination of the new wafer stored by the FOUP already contamined by the volatile acid.

Indeed, in order to successfully ensure the miniaturization the integrated circuits manufacturing, we have mainly through the advent of $300[\mathrm{~mm}]$ wafer manufacturing technologies new methods that are required for facing this challenge. To endeavor a systematic analysis and control of the underlying system, numerical simulation should help to mimic the process behaviors. Modeling and computational method are worth method to predict and to quantify physical phenomenon such as AMCs cross contamination within FOUP.

Motivated by the above phenomena, this work describes and develops the finite element formulation devoted to AMCs cross contamination in order to investigate the effect of the contaminant to its close environment. To propose new strong numerical tools for AMCs cross contamination able to qualify and to quantify the residual contamination in the FOUP. The model validation method is based on correlation of the observed data and the direct method together.

\section{Model settings}

Modeling the adsorption of AMCs cross contamination between wafer and FOUPs is based on adsorption phenomena. In general two concepts of adsorption, physical and chemistry adsorption models will be considered. A detailed review of various adsorption kinetics (adsorption, desorption) models was given in [6]-[7]. In this model the transport towards the surface is purely diffusive and we investigate the concentration in the internal area of FOUPs by using the thermodynamics laws. These thermodynamics laws are traduced by the Henry constant in order to connect the concentration of the contaminant at the FOUPs atmosphere and the concentration at the internal surface of the FOUPs.

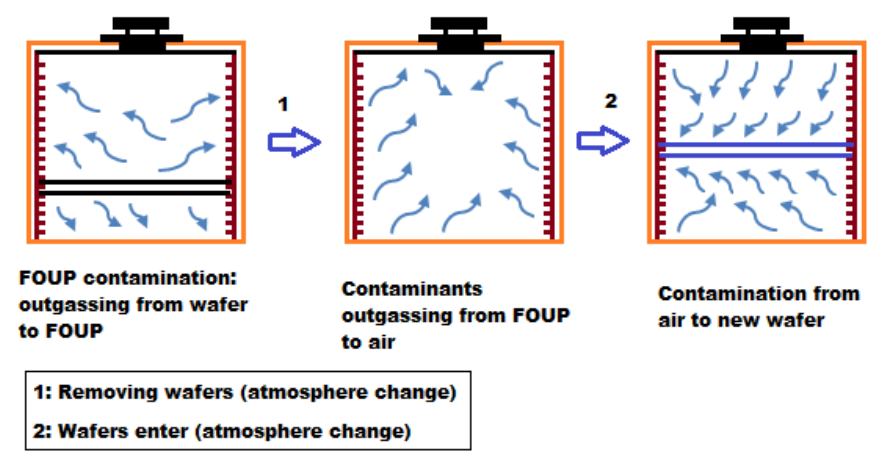

Fig. 1: Schematic Illustration for the Three Main Cross Contamination Processes Steps.

The mathematical model of diffusion process in these two domains is based on Fick's second law of diffusion [8]. At the interface of that domain a kinetics law will be established. According to Fick's first and second law, also known as the diffusion equation [9]-[10], is defined in the first part of the equation (1).

Where here $\mathrm{i}:=\mathrm{s}, \mathrm{g}$. The AMCs cross contamination is governed by diffusion time dependent process in which the rate of diffusion is a fraction of time. In this process, the contaminant moves from a region of high concentration (wafer) to one of low concentration (internal surface of FOUP) [11].

According to Fick's first and second law, also known as the diffusion equation [10] also known as the diffusion equation, the flux per unit of area perpendicular to the flux direction is given by:

$\mathrm{J}_{\mathrm{i}}=-\mathrm{D}_{\mathrm{i}} \nabla \mathrm{C}^{\mathrm{i}}$ 
Thus the mathematical expression for transient contaminant transfer between the wafer and the internal part of the FOUP is given by the convection-diffusion equation (2). This equation traduced the conservation equation for the species $\mathrm{i}$ and its can be written:

$\frac{\partial \mathrm{C}^{\mathrm{i}}}{\partial \mathrm{t}}=\nabla \cdot\left(\mathrm{D}_{\mathrm{i}} \nabla \mathrm{C}^{\mathrm{i}}\right)-\nabla\left(\mathrm{u} \mathrm{C}^{\mathrm{i}}\right)+\mathrm{q}$

The contaminant transfer between the internal parts to the polymer (FOUP) is purely diffusive, and we have

$\frac{\partial C^{\mathrm{i}}}{\partial \mathrm{t}}=\nabla \cdot\left(\mathrm{D}_{\mathrm{i}} \nabla \mathrm{C}^{\mathrm{i}}\right)+\mathrm{q}$

Were here $\mathrm{i}$ is the species.

The AMCs cross contamination is governed by the diffusion with time dependent process in which the rate of diffusion is function of time. In this process, contaminant moves from a region of high concentration (wafer post processed) to one of low concentration (internal surface of FOUP). In the outgassing, phenomena the contaminant moves from FOUP to the wafer. We consider that, on $\Gamma_{\mathrm{D}}$, we have the wafer area, in which the source of contamination takes place during the contamination time $t_{c}$.

In the outgassing phenomenon, the contaminant moves from FOUP to the wafer. We assume that, on, we have the wafer area in which the source of contamination takes place during the contamination time. We assume that the advection and reaction time scales are slow compared to the diffusive time scales. The equation for transient contaminant transfer between the wafer and the internal part of the FOUP is given by (4):

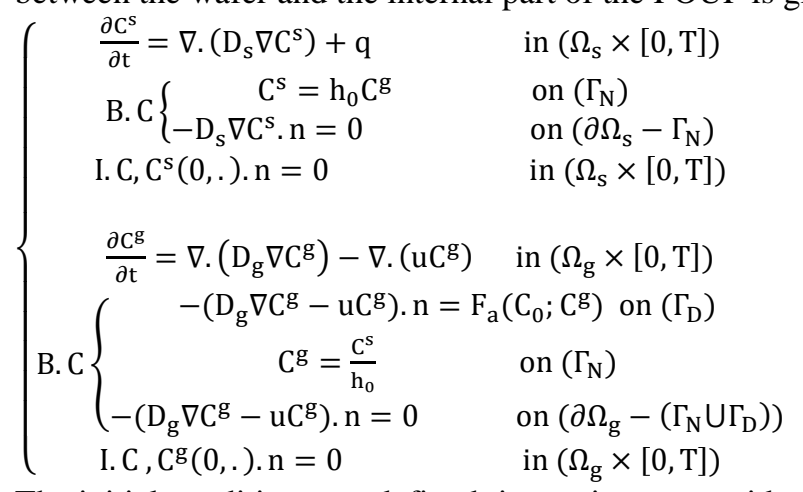

The initial conditions are defined; it consists to consider that at the initial time $t:=0$ the FOUP and its atmosphere are not yet contamined i.e. $\mathrm{C}^{\mathrm{S}}(\mathrm{t}=0):=0$ at $\mathrm{C}^{\mathrm{g}}(\mathrm{t}=0):=0$. Practically, these studies have done with a new FOUP for a first use. In the model, $\mathrm{D}_{\mathrm{g}}$ and $\mathrm{D}_{\mathrm{s}}$ are the gas diffusion coefficient in gas and solid medium, which has no connection with the spatial location and no variation in time, $\left[\mathrm{m}^{2} / \mathrm{s}\right]$. The unknown of the problem are $\mathrm{C}^{\mathrm{g}}$ and $\mathrm{C}^{\mathrm{s}}$ which are respectively the concentration of the contaminant and the concentration at the internal FOUP's surface $H(t-\varepsilon)$ is the Heaviside function, $\mathrm{C}_{0}$ is the initial concentration in FOUP's atmosphere when the wafers have been finished to outgassing the contaminant, $\mathrm{h}_{0}$ is the Henry constant and $\mathrm{n}$ is the outer unit normal vector, $\mathrm{T}$ is the final time and $\mathrm{q}$ is the source.

Because the wall concentration $\mathrm{C}^{\mathrm{s}}$ depends on the concentration $\mathrm{C}^{\mathrm{g}}$ we assume the following boundary condition: on $\Gamma_{\mathrm{D}}$ a prescribed inflow concentration is imposed and at the interface the two concentrations will be balanced by the Henry constant. We start by developing some analytical solutions for the diffusion equation with uniform diffusivities in an unbounded domain and for very simple boundary conditions as determined by J. Crank. Then we solve the more general equation using finite element method, a numerical technique of optimization, for any type of boundary conditions. In this approach, we use the assumptions below. We suppose that, the FOUP and the membrane are homogenous and isotropic. Adsorption resolution of the contaminant is balanced at the surface [12], [13]. The surface roughness of the internal surface of the FOUP or the membrane is neglected but we take into account of this parameter when we use the Henry law at the surface. Then the diffusion coefficient is weakly function of the concentration.

$\mathrm{F}_{\mathrm{a}}\left(\mathrm{C}_{0} ; \mathrm{C}^{\mathrm{g}}\right)=\mathrm{N}_{0}^{\mathrm{g}}+\mathrm{k}_{\mathrm{c}}\left[\mathrm{C}_{0} \mathrm{H}(\mathrm{t}-\varepsilon)-\mathrm{C}^{\mathrm{g}}\right]$

We need to switch $-\left(D_{g} \nabla C^{g}+u^{g}\right) \cdot n=F_{a}\left(C_{0} ; C^{g}\right)$ to $-\left(D_{g} \nabla C^{g}+u C^{g}\right) \cdot n=0$ during the simulation.

Firstly we assume that there is no inner flux $\mathrm{N}_{0}^{\mathrm{g}}$ (no initial flux) and we assume that we have a transient boundary conditions with a laminar gas flow on this boundary $(\mathrm{u}=0)$. When $\mathrm{k}_{\mathrm{c}}$ is sufficiently large, we have the Dirichlet condition i.e $\mathrm{C}^{\mathrm{g}} \simeq \mathrm{C}_{0} \mathrm{H}(\mathrm{t}-\varepsilon)$ and if $\mathrm{k}_{\mathrm{c}}$ we have the Neumann's boundary condition (for the neutral area of the wafer), i.e. $\left(-D_{g} \nabla C^{g}+u C^{g}\right) \cdot n=0$. To conditionning $C_{0}$ and $k_{c}$ we need a parametric study was made to ensure the stability. We have,

if $\left\{\begin{array}{c}\mathrm{k}_{\mathrm{c}} \geq 1 \Rightarrow \mathrm{C}^{\mathrm{g}} \simeq \mathrm{C}_{0} \mathrm{H}(\mathrm{t}-\varepsilon) \text { if } 0<t \leq\left(\mathrm{t}_{\mathrm{c}}+\mathrm{t}_{\mathrm{p}}\right) \\ \mathrm{k}_{\mathrm{c}}=0 \Rightarrow\left(-\mathrm{D}_{\mathrm{g}} \nabla \mathrm{C}^{\mathrm{g}}+\mathrm{uC}^{\mathrm{g}}\right) \cdot \mathrm{n}=\mathrm{N}_{0}^{\mathrm{g}} \text { if } \mathrm{t}>\left(\mathrm{t}_{\mathrm{c}}+\mathrm{t}_{\mathrm{p}}\right)\end{array}\right.$

Where the computed quantity is obtained by the analytical solution, such that the concentration at the internal surface is $\mathrm{Q}_{\infty}$ and there is no initial distribution in the polymer membrane. The diffusion coefficient $\mathrm{D}$ is the unknown; it is obtained by using the optimization method. 
The Fick's law involves the diffusion coefficient of contaminant through the polymeric material. However, the literature doesn't provide enough data concerning the molecular diffusion. In order to find the values of the diffusion coefficients, a numerical optimization are established by using the experiment data.

This method is used to calculate the diffusion coefficient for each contaminant in the polymer material constituent of the FOUP. It does consist to minimize the equation (7) which fits the diffusion coefficient as parameters of the model function to experimental data of the sorbed quantity-time curves.

According to J. Crank [14] the amount of contaminant is a parametric function of time and diffusion coefficient, for a membrane with thickness $L / 2$ :

$Q\left(t, D_{S}\right)=Q_{\infty}\left[1-\sum_{n=0}^{\infty} \frac{8}{(2 n+1)^{2} \pi^{2}} \exp \left(-\frac{D_{S}(2 n+1)^{2} \pi^{2} t}{4 L^{2}}\right)\right]$

We use the nonlinear least square to determine the diffusion coefficient for each contaminant in the polymer membrane, the as objective function using the experimental data and according to Urruty and al. method in [15]-[16], can be written $f(x)=\frac{1}{2}\left\|Q^{\exp }-Q^{\text {the }}\right\|^{2}$

where $Q^{\exp }$ and $Q^{\text {the }}$ are the experimental quantity of the contaminant recorded inside the polymer membrane (FOUP) and the amount of incorporated contaminant is computed using the analytical solution defines at the end of the section 3.2. According the matrix notation the equation (8) becomes:

$f(D)=\frac{1}{2} \sum_{i=1}^{m}\left[Q_{i}^{\text {exp }}-Q_{i}^{\text {the }}\left(t_{i} ; D\right)\right]^{2}=\frac{1}{2} \sum_{i=1}^{m} r^{2}(D)$

In this expression, the diffusion coefficient $\mathrm{D}$ is the unknown. In order to determine $\mathrm{D}$, we have to minimize the objective function given by

$\min _{D} f(D)=\min \frac{1}{D} \sum_{i=1}^{m}\left[Q_{i}^{\exp }-Q_{\infty}\left[1-\sum_{n=0}^{\infty} \frac{8}{(2 n+1)^{2} \pi^{2}} \exp \left(-\frac{D(2 n+1)^{2} \pi^{2} t_{i}}{4 L^{2}}\right)\right]\right]^{2}$

This function has one global minimum and the set the diffusion coefficient $\mathrm{D}$ which belongs to this minimum is defined to be optimal fitting to the experimental data.

In order to evaluate the correlation between the data and model we need to evaluate the coefficient of determination. It measures how well the regression line represents the data.

$0 \leq R^{2}=1-\frac{\|r\|^{2}}{\left\|Q^{e x p}-\overline{Q^{t h e}} \mathbb{I}\right\|^{2}} \leq 1$

\subsection{Contamination process}

We start directly from the contamination event, when the wafers processes were finished, it will be stored in the FOUP. These wafers are contaminated by the volatile acid caused by the process: the chemical product used in wafer processing is the main sources of this AMC. In the FOUP the wafers outgases this volatile acid during a few minutes or hours, it depends of the contamination level. And after that, the outgassing step is completed but the contaminant continues to move from air to the internal surface of the FOUP and follow by contaminant diffusion in the polymer.

In practice of experimental imitation of the contamination process the contaminant is intentionally introduced in the FOUP. This experimental procedure allow to hold steady the concentration of contaminant close to 1000 [ppbv] in the FOUP.

This technique mimics the process during which the wafer outgassing the pollutant which contaminates the FOUP. This is the first step of the cross contamination. The pollutant moves from wafer to FOUPs. During this process, the surface adsorption step takes place and the diffusion in the volume of the FOUP polymeric materials happens. Contamination simulations consist to use the same conditions and assumptions which have been developed in equation (6). Thus, we consider that the wafer is the contaminant source governed by the Heaviside function with a delay $\varepsilon$. The amplitude of the contamination on the wafer is $C_{0}$. The implementation of this boundary condition is defined in equation (6). Therefore, during $t_{c}$ (contamination time), we apply in $\Gamma_{D}$ (wafer's surface) the concentration $C_{0}$ governed by the Heaviside function, after $t_{c}=\widetilde{t}_{c}+t_{0}$ the wafer surface stops to outgas the contaminant and stays neutral.

$\left\{\begin{array}{rr}F_{a}\left(C_{0} ; C^{g}\right)=N_{0}^{g}+k_{c}\left[C_{0} H(t-\varepsilon)-\overline{\left.C^{g}\right]}\right. & \text { lf } 0<t \leq \tilde{t}_{c} \\ F_{a}\left(C_{0} ; C^{g}\right)=0 & \text { lf } \tilde{t}_{c}<t \leq\left(\tilde{t}_{c}+t_{0}\right)\end{array}\right.$

The experiment process prescribe that the contamination time $t_{c}$ is decomposed in two characteristic time $\tilde{t}_{c}$ and $t_{0}$ respectively the time until the wafer outgassing is completed and the downtime before opening the FOUP to remove the wafer The implementation of the boundary conditions are defined in the equation (6), that was the same method validated in part I.

When $t \leq \tilde{t}_{c}$ the boundary condition describes (cf.eq.6) the wafer post processed outgassing the contaminant. And the second boundary condition $\left(\tilde{t}_{c}<t \leq\left(\tilde{t}_{c}+t_{0}\right)\right.$ ) defined the condition during which the wafer outgassing is done but it was stored in the FOUP (i.e. we consider that the wafer surface become neutral).

\subsection{Purging and outgassing processes}

This procedure takes place after opening the FOUP in the goal to remove the wafer post processed. When the door of the FOUP is opened, airflow from the exterior atmosphere go into and modify the contaminant concentration due to the dilution of the FOUP atmosphere by room air. An inert gas from exterior atmosphere is supplied on their pods. 
Applying an inert gas purge in FOUPs will be doing with an inlet and outlet ports on the pods and with the interface ports. Nevertheless during this process we consider only the removing atmosphere during which the front door is open. The FOUP atmosphere is removed and the contaminant concentration $C^{g} \simeq 0$ during this operation before it the front door is closed. Its results a reverse flow of the contamination gradient during which the contaminant moves from FOUP materials (from polymeric materials to air and from air to wafer). After the contamination of the FOUP $t_{c}$ the FOUP has been opened during the opening time $t_{p}$ then the FOUP has been closed during the outgassing time $t_{d}$.

$\left\{\begin{aligned} F_{a}\left(C_{0} ; C^{g}\right)= & N_{0}^{g}+k_{c}\left[0 . H \overline{(t-\varepsilon)}-C^{g}\right] \\ F_{a}\left(C_{0} ; C^{g}\right)= & 0 \quad \text { if } t_{c}<t \leq\left(t_{c}+t_{p}\right) \\ & \text { lf }\left(t_{c}+t_{p}\right)<t \leq\left(t_{c}+t_{p}+t_{d}\right)\end{aligned}\right.$

When $t_{c}<t \leq\left(t_{c}+t_{p}\right)$ (3) boundary condition mimics the opening door of the FOUP the wafer moves and the removing atmosphere. Experimentally, the measurement during the opening door processes exhibited that the contaminant concentration at the atmosphere is negligible and close to zero. Therefore we this concentration (zero) is applied during this operation and again the surface become neutral after closing door.

\subsection{Decontamination and cleaning process}

In this section, we describe the application of the model to the decontamination process. This operation consists to introduce a downward flow of inert gas in order to clean up the FOUP. Many process of purge are considered and are studied by theoretical analysis and experimental simulation and measurement. Purging FOUP with inert gas is the one of the most popular method, but there are many several methods as UV or vacuum method. During purging the amount of the inert gas can evaluate as function of temperature process in the goal to eliminate any undesirable contamination. Therefore it is possible to estimate the mean value of velocity of the inert gas flow. By using the present model various values of velocity could be applied on the FOUP process decontamination.

Subsequently outgassing, the decontamination could be done. Decontamination is the processes of removing contaminant that have accumulated inside polymeric materials of the FOUPs. Decontamination is employed in order to reduce the AMCs cross contamination. In this work, we consider two types of procedures, the cold purging and the warm purging. In the goal of this section is to study the temperature effect during the FOUPs cleaning. In general purging the FOUP with inert gas provides the many advantages. The decontamination process will be done during $t_{u}$ we denote $t_{w}$ the downtime when the FOUP is closed after decontamination process. This waiting time is important to known the cleaning efficiency. So the final time $T$ of the simulation is the sum of all characteristic time $t_{i}$, it can be written:

$T=\sum_{i} t_{i}=t_{c}+t_{p}+t_{d}+t_{u}+t_{w}$

Ended, the total time is defined as $t \in\left[0, \sum_{i} t_{i}\right]$ or $t \in[0, T]$

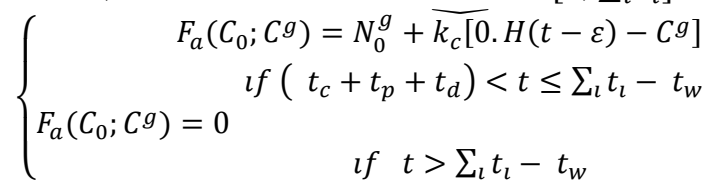

The first boundary condition (15) represents the cleaning operation of the FOUP by purging with inert gas. Experimentally, an amount of the inert gas was introduced in the FOUP. The present assumption gauges that the contaminant concentration at the atmosphere is negligible and close to zero. Indeed $t$ during the operation the equation (15) describes the cold cleaning operation i.e. the cleaning operation is done with the ambient temperature (in general $\left.25^{\mathrm{O}} \mathrm{C}\right)$.

In order to evaluate the effect of the temperature with the cleaning operation, the Arrhenius law is given by the equation (16). Is used this law gives the relation between the diffusion coefficient at the reference temperature and the diffusion coefficient at the temperature $T$. Thus the diffusion coefficient could be expressed as function of temperature and it is possible to use the same boundary condition. The diffusion coefficient change with the temperature is given by:

$D_{s, g}=D_{s, g}^{0} \exp \left(-\frac{E_{0}^{s, g}}{R T^{s, g}}\right)$

Where $D_{s, g}^{0}$ is the diffusion coefficient at the reference temperature a. $E_{0}^{s, g}$ is the free enthalpy of the contaminant in gas phase or in the polymer state. $T^{s, g}$ And $R$ is respectively the operating temperature in each phases and the perfect gas constant. Practically, the purging temperature during the "hot purging" is continuous in both phases, and we introduce this temperature in the Arrhenius law.

\subsection{Finite element discretization}

A table, we use the Galerkin finite element formulation [17], [18] for numerical solutions of the problem given by equation (1). It is obtained by multiplying the equilibrium equation by an appropriate test function respectively $\phi$ and $\psi$ for the concentration $C^{s}$ and $C^{g}$ respectively, and by integrating over the computational domain. In this case, we have 
$\left\{\begin{array}{c}\int_{\Omega_{s}} \frac{\partial C^{s}}{\partial t} \phi d V=\int_{\Omega_{s}} \nabla \cdot\left(D_{s} \nabla C^{s}\right) \phi d V+\int_{\Omega_{s}} q d V \\ \int_{\Omega_{g}} \frac{\partial C^{g}}{\partial t} \psi d V=\int_{\Omega_{g}} \nabla \cdot\left(D_{s} \nabla C^{g}\right) \psi d V-\int_{\Omega_{s}} \nabla\left(u C^{g}\right) \psi d V\end{array}\right.$

For that, let us consider first the functional $H^{1}\left(\Omega_{s}\right)$ and $H^{1}\left(\Omega_{g}\right)$ in which we search the solution in accordance with regularity $H_{s}^{1}=\left\{\phi \in H^{1}\left(\Omega_{s}\right) \mid \phi=s \forall x \in \Gamma_{s}\right\}$ and $H_{s}^{1}=\left\{\psi \in H^{1}\left(\Omega_{s}\right) \mid \psi=s \forall x \in \Gamma_{g}\right\}$ where $H_{s}^{1}\left(\Omega_{s}\right)$ and $H_{s}^{1}\left(\Omega_{g}\right)$ are a Sobolev space, classically defined as $H_{s}^{1}\left(\Omega_{s}\right)=\left\{\phi \in L^{2}\left(\Omega_{s}\right),\|\nabla \phi\| \in L^{2}\left(\Omega_{s}\right)\right\}$ and $H_{s}^{1}\left(\Omega_{g}\right)=\left\{\psi \in L^{2}\left(\Omega_{g}\right),\|\nabla \psi\| \in\right.$ $\left.L^{2}\left(\Omega_{g}\right)\right\}$. Where $L^{2}\left(\Omega_{s}\right)$ and $L^{2}\left(\Omega_{g}\right)$ are the Hilbert vector space of the functions quadrically summable respectively on $\left(\Omega_{s}\right)$ and $\left(\Omega_{g}\right)$.

Therefore, $L^{2}\left(\Omega_{s}\right)=\left\{\left.\phi(x)\left|\int_{\Omega_{s}}\right| \phi(x)\right|^{2} d x<\infty\right\}$ and $L^{2}\left(\Omega_{g}\right)=\left\{\left.\psi(x)\left|\int_{\Omega_{g}}\right| \psi(x)\right|^{2} d x<\infty\right\}$ are considered.

By using Green's theorem [18], integration by parts leads to,

$\left\{\begin{array}{c}\int_{\Omega_{s}} \frac{\partial C^{s}}{\partial t} \phi d V=-\int_{\Omega_{s}}\left(D_{s} \nabla C^{s}\right) \cdot \nabla \phi d V+\int_{\Omega_{s}}\left(D_{s} \nabla C^{s}\right) \cdot n \phi d V+\int_{\Omega_{s}} q \phi d V \\ \quad \int_{\Omega_{g}} \frac{\partial C^{g}}{\partial t} \psi d V=-\int_{\Omega_{s}}\left(D_{g} \nabla C^{g}\right) \cdot \nabla \psi d V+\int_{\Omega_{s}}\left(D_{g} \nabla C^{g}\right) \cdot n \psi d V-\int_{\Omega_{s}} \nabla\left(u C^{g}\right) \psi d V\end{array}\right.$

By applying the Galerkin's weighted residual method and the Green's theorem mentioned above, the variational formulation corresponding the AMCs cross contamination is given by the equation (17). Consequently the weak formulation of the AMCs model should be introduced:

We find $C^{s} \in H^{1}\left(\Omega_{s}\right)$ and $C^{g} \in H^{1}\left(\Omega_{g}\right)$ such that,

$\left\{\begin{array}{l}a_{1}\left(\frac{\partial C^{s}}{\partial t}, \phi\right)+b_{1}\left(C^{s}, \phi\right)=L_{1}(\phi) \forall \phi \in H^{1}\left(\Omega_{s}\right) \\ a_{2}\left(\frac{\partial C^{g}}{\partial t}, \psi\right)+b_{2}\left(C^{g}, \psi\right)=L_{2}(\psi) \forall \psi \in H^{1}\left(\Omega_{g}\right)\end{array}\right.$

$L_{1}(\phi)=\int_{\Omega_{s}} q \phi d V$

$L_{2}(\phi)=\int_{\Omega_{s}} N_{0}^{g} \psi d V$

$a_{1}\left(\frac{\partial C^{s}}{\partial t}, \phi\right)=\int_{\Omega_{s}} \frac{\partial C^{s}}{\partial t} \phi d V$

$a_{2}\left(\frac{\partial C^{g}}{\partial t}, \psi\right)=\int_{\Omega_{g}} \frac{\partial C^{g}}{\partial t} \psi d V$

$b_{1}\left(C^{s}, \phi\right)=-\int_{\Omega_{s}}\left(D_{s} \nabla C^{s}\right) \cdot \nabla \phi d V$

$b_{2}\left(C^{g}, \phi\right)=\int_{\Omega_{s}}\left(D_{g} \nabla C^{g}\right) \cdot \nabla \psi d V-\int_{\Omega_{s}} \nabla\left(u C^{g}\right) \psi d V+\int_{\Gamma_{D}} F_{a} \psi d S$

With the conditions defined follow:

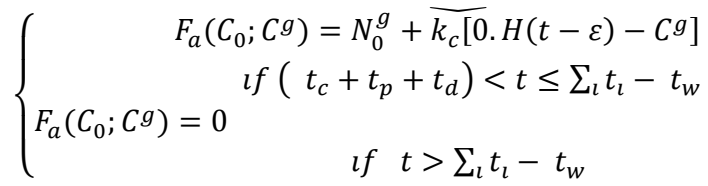

Notice that the domain $\Omega_{s}$ and $\Omega_{g}$ are decomposed into finite subdomains $\Omega_{s}^{e}$ and $\Omega_{g}^{e}$ for each elements. Similarly, boundary $\partial \Omega_{s}$ and $\partial \Omega_{g}$ are decomposed in $\partial \Omega_{s}^{e}$ and $\partial \Omega_{g}^{e}$. Finally, the time interval is subdivided by $n$ subintervals.

For the spatial discretization, we consider the finite element partition $\mathcal{T}_{h}^{s}$ and $\mathcal{T}_{h}^{s}$ of $\Omega_{s}$ and $\Omega_{g}$ respectively into tetrahedral elements. Again for simplicity, we will considerthat the finiteelement partition associated to $\mathcal{T}_{h}^{S}$ and $\mathcal{T}_{h}^{s}$ are uniform, $h$ is the size of the element domains. Let us $C_{h}^{s}$ and $C_{h}^{g}$ are the approximations solutions of $C^{s}$ and $C^{g}$ respectively.

The Galerkin's approximation above becomes:

We find $C_{h}^{s} \in H^{1 h}\left(\Omega_{S}\right)$ and $C_{h}^{g} \in H^{1 h}\left(\Omega_{g}\right)$

$$
\left\{\begin{array}{l}
a_{1}\left(\frac{\partial C_{h}^{s}}{\partial t}, \phi_{h}\right)+b_{1}\left(C_{h}^{s}, \phi_{h}\right)=L_{1}\left(\phi_{h}\right) \forall \phi_{h} \in H^{1 h}\left(\Omega_{s}\right) \\
a_{2}\left(\frac{\partial C_{h}^{g}}{\partial t}, \psi_{h}\right)+b_{2}\left(C_{h}^{g}, \psi_{h}\right)=L_{2}\left(\psi_{h}\right) \forall \psi_{h} \in H^{1 h}\left(\Omega_{g}\right)
\end{array}\right.
$$

Finally, we obtain a system of first order differential equations and using the matricial notation, we have

$\left[\begin{array}{cc}\mathbb{M}^{s} & 0 \\ 0 & \mathbb{M}^{g}\end{array}\right]\left(\begin{array}{c}\dot{C}^{s} \\ \dot{C}^{g}\end{array}\right)+\left[\begin{array}{cc}\mathbb{D}^{s} & h_{0} \\ h_{0}^{-1} & \mathbb{D}^{g}\end{array}\right]\left(\begin{array}{c}C^{s} \\ C^{g}\end{array}\right)=\left(\begin{array}{l}\mathbb{F}^{s} \\ \mathbb{F}^{g}\end{array}\right)$

Where $C^{s}$ and $C^{g}$ are the vectors of nodal unknown concentrations, $\mathbb{M}^{s}$ and $\mathbb{M}^{g}$ are the time constant matrices, $\mathbb{F}^{s}$ and $\mathbb{F}^{g}$ are the source and external flux vector. The system of ordinary differential equations above has to be integrated in time. Using the finite difference approximations, explicit Euler scheme, and the system can be written at time $t+\Delta t$ as: 
$\left[\begin{array}{cc}\mathbb{M}^{s} & 0 \\ 0 & \mathbb{M}^{g}\end{array}\right]^{e}\left(\begin{array}{l}C_{n+1}^{s}-C_{n}^{s} \\ C_{n+1}^{g}-C_{n}^{g}\end{array}\right)^{e}+\Delta t\left[\begin{array}{cc}\mathbb{D}^{s} & h_{0} \\ h_{0}^{-1} & \mathbb{D}^{g}\end{array}\right]^{e}\left(\begin{array}{l}C_{n}^{s} \\ C^{g}\end{array}\right)^{e}=\Delta t\left(\begin{array}{l}\mathbb{F}_{n}^{s} \\ \mathbb{F}_{n}^{g}\end{array}\right)^{e}$

Where,

$\mathbb{F}_{n}^{s}=\int_{\Omega_{s}^{e}} q N d V$

$\mathbb{F}_{n}^{g}=\int_{\Omega_{g}^{e}} N_{0}^{g} N d V$

$\mathbb{M}^{S}=\int_{\Omega_{s}^{e}} N^{T} N d V$

$\mathbb{M}^{g}=\int_{\Omega_{g}^{e}} N^{T} N d V$

$\mathbb{D}^{S}=\int_{\Omega_{s}^{e}} D_{s} \nabla N^{T} \cdot N d V$

$\mathbb{D}^{g}=\int_{\Omega_{g}^{e}} D_{g} \nabla N^{T} \cdot N d V+\int_{\Omega_{g}^{e}} \nabla \cdot\left(u N^{T}\right) d V+\int_{\Gamma_{D}} F_{a}\left(N^{T}\right) N d S$

Also with:

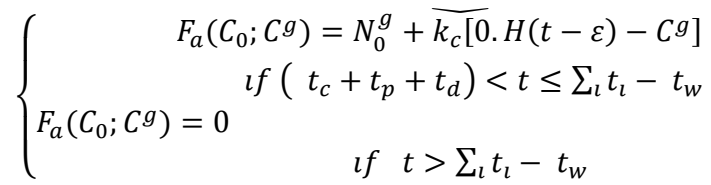

And $N$ denote the linear interpolation function at each bode. By using the assembly theory for all subdomains, we have:

$\mathrm{U}_{e=1}^{m}\left[\begin{array}{cc}\widetilde{\mathbb{M}}^{s} & 0 \\ 0 & \widetilde{\mathbb{M}}^{g}\end{array}\right]^{e}\left(\begin{array}{c}C_{n+1}^{s}-C_{n}^{s} \\ C_{n+1}^{g}-C_{n}^{g}\end{array}\right)^{e}+\cup_{e=1}^{m} \Delta t\left[\begin{array}{cc}\widetilde{\mathbb{D}}^{s} & h_{0} \\ h_{0}^{-1} & \widetilde{\mathbb{D}}^{g}\end{array}\right]^{e}\left(\begin{array}{c}C_{n}^{s} \\ C^{g}\end{array}\right)^{e}=\cup_{e=1}^{m} \Delta t\left(\begin{array}{c}\widetilde{\mathbb{F}}_{n}^{s} \\ \widetilde{\mathbb{F}}_{n}^{g}\end{array}\right)^{e}$

Where

$\widetilde{\mathbb{M}}_{i j}^{s, g}=\sum_{i j}^{p_{-} \text {nodes }} \mathbb{M}_{i j}^{s, g}, \widetilde{\mathbb{D}}_{i j}^{s, g}=\sum_{i j}^{p_{\text {nodes }}} \mathbb{D}_{i j}^{s, g}, \widetilde{\mathbb{F}}_{j}^{s, g}=\sum_{j}^{p_{\text {nnodes }}} \mathbb{F}_{j}^{s, g}$

By considering this switch conditions, the matrix components can be written as:

$\mathbb{F}_{n}^{s}=\int_{\Omega_{s}^{e}} q N d V$

$\mathbb{F}_{n}^{g}=\int_{\Omega_{g}^{e}} N_{0}^{g} N d V$

$\mathbb{M}^{S}=\int_{\Omega_{s}^{e}} N^{T} N d V$

$\mathbb{M}^{g}=\int_{\Omega_{g}^{e}} N^{T} N d V$

$\mathbb{D}^{S}=\int_{\Omega_{\mathrm{s}}^{e}} D_{S} \nabla N^{T} \cdot N d V$

$\mathbb{D}^{g}=\int_{\Omega_{g}^{e}} D_{g} \nabla N^{T} \cdot N d V+\int_{\Omega_{g}^{e}} \nabla \cdot\left(u N^{T}\right) d V+\int_{\Gamma_{D}} F_{a}\left(N^{T}\right) N d S$

And then,

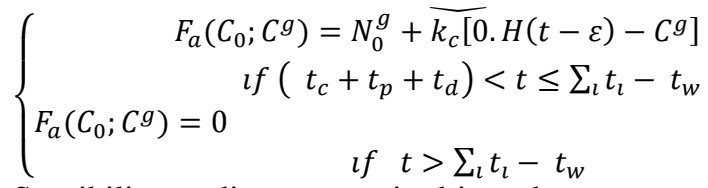

Sensibility studies are required in order to gauge if an existing method or model (particularly the boundary conditions defined above) is optimal, correct, stable or not. Indeed, we have scrutinized the effect of varying value of parameters (Henry constant, diffusion coefficient, maximum concentration on the wafer surface) as function of time.

The first condition is: there is no more than one solution.

We have:

$\left\|C_{h}^{g, n}\right\|_{0} \leq\left\|C_{h}^{g, 0}\right\|_{0}+T\left\|L_{2}\right\|_{0}+\frac{T}{2} D_{g}\left\|\nabla \cdot\left(u L_{2}\right)\right\|_{0}$

$\left\|C_{h}^{g, n}-C(n \Delta t)\right\|_{0} \leq c\left(h^{2}+\Delta t^{2}\right)$

$\left\|C_{h}^{g, n}\right\|_{0}^{2}+\frac{\Delta t^{2}}{2}\left\|\nabla \cdot\left(u C_{h}^{g}\right)\right\|^{2}+D_{g}\left\|\nabla C_{h}^{g, n}\right\|_{0}^{2}$

A small change in the equation or in the side, initial and/or boundary conditions, gives rise to a small change in the solution.

$\left\|C_{h}^{g, n+1}\right\|_{0}^{2}+\frac{\Delta t^{2}}{2}\left\|\nabla \cdot\left(u C_{h}^{g, n+1}\right)\right\|^{2}+D_{g}\left\|\nabla C_{h}^{g, n+1}\right\|_{0}^{2} \leq$ 
$\left\|C_{h}^{g, n+1}\right\|_{0}\left\|C_{h}^{g, n}\right\|_{0}+\Delta t\left\|C_{h}^{g, n+1}\right\|_{0}\left\|L_{2}\right\|_{0}+\frac{\Delta t^{2}}{2}\left\|\nabla .\left(u C_{h}^{g}\right)\right\|_{0}\left\|C_{\mathrm{h}}^{\mathrm{g}, \mathrm{n}+1}\right\|_{0}$

Dividing (22) by $\left\|\mathrm{C}_{\mathrm{h}}^{\mathrm{g}, \mathrm{n}+1}\right\|_{0}$, we have

$\left\|\mathrm{C}_{\mathrm{h}}^{\mathrm{g}, \mathrm{n}}\right\|_{0} \leq\left\|\mathrm{C}_{\mathrm{h}}^{\mathrm{g}, 0}\right\|_{0}+\mathrm{T}\left\|\mathrm{L}_{2}\right\|_{0}+\frac{\mathrm{T}}{2} \mathrm{D}_{\mathrm{g}}\left\|\nabla .\left(\mathrm{uL}_{2}\right)\right\|_{0}$

The convergence is the sum of the consistence (precision) and the stability. When the model is stable the solution should be compared with the experimental data in order to ensure the consistence of the model.

\section{Main results and discussions}

In this section, we present the validation of the model with experiment data. We discuss many conditions corresponding to the industrial's application (contamination process, opening and purging process). In practice, this method is defined as the contaminant concentration increase to the maximum value during the contamination time, and when the contamination time is finished, the wafer area is considered as a neutral area.
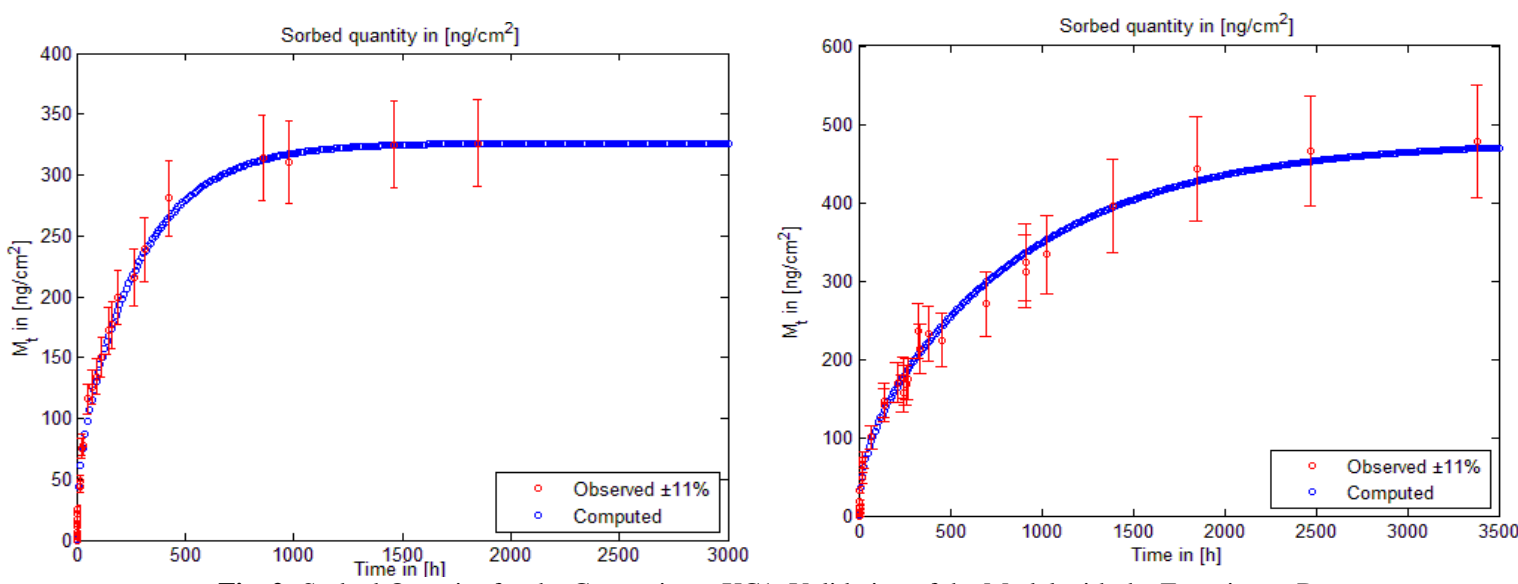

Fig. 2: Sorbed Quantity for the Contaminant XC1: Validation of the Model with the Experiment Data.

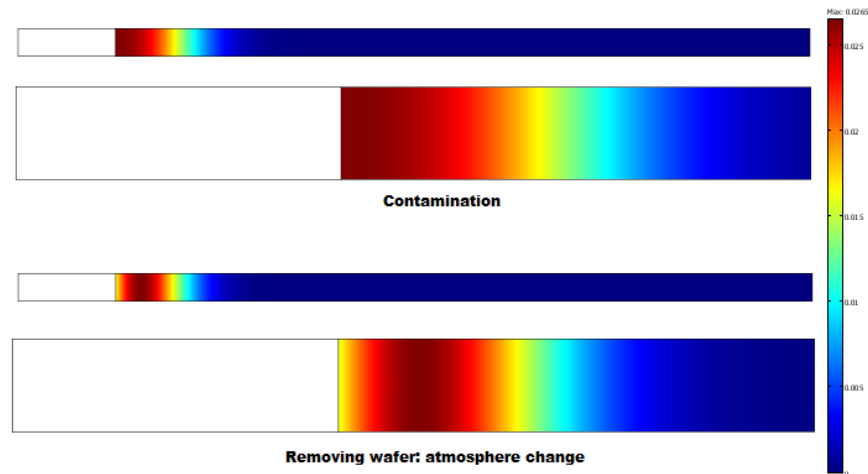

Fig. 3: Outgassing Process after Removing FOUP, Contaminant Moves From FOUP's Surface to Atmosphere.
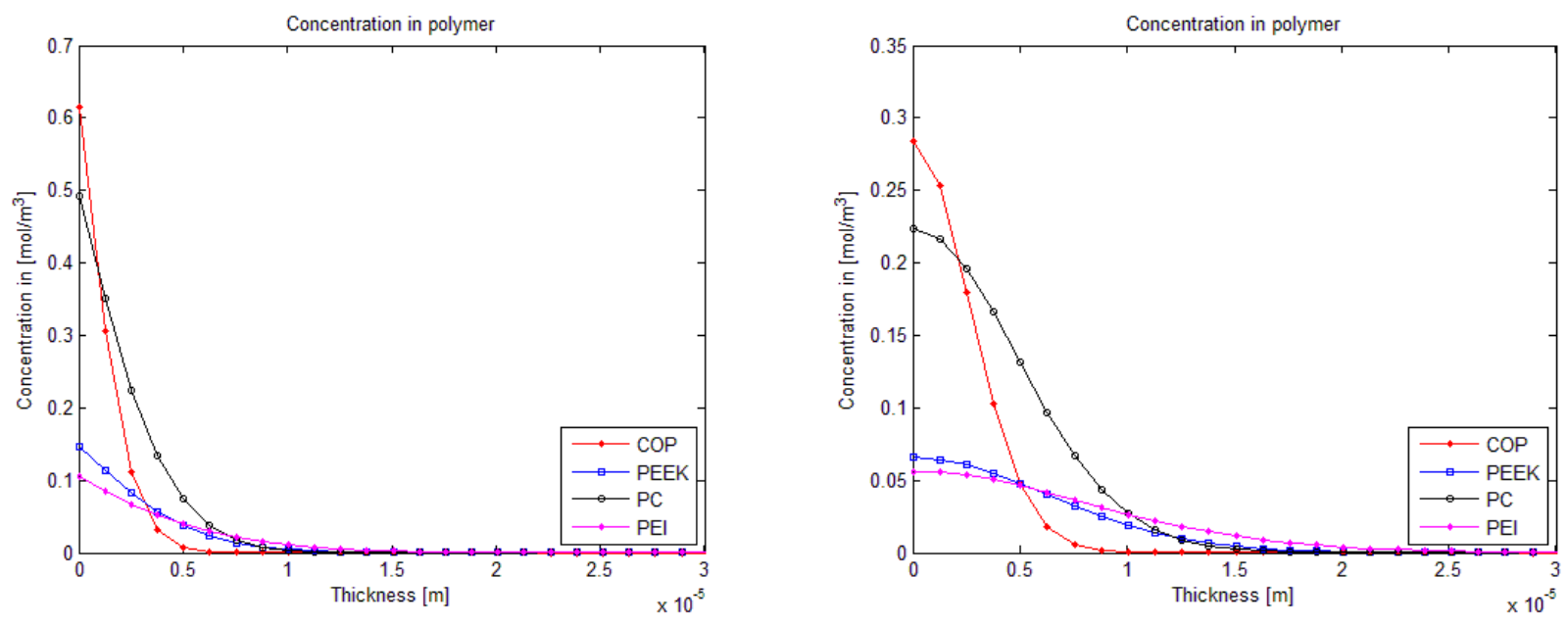

Fig. 4: Concentration in Each Material (Polymer) By Using the Same Conditions in Contamination. 

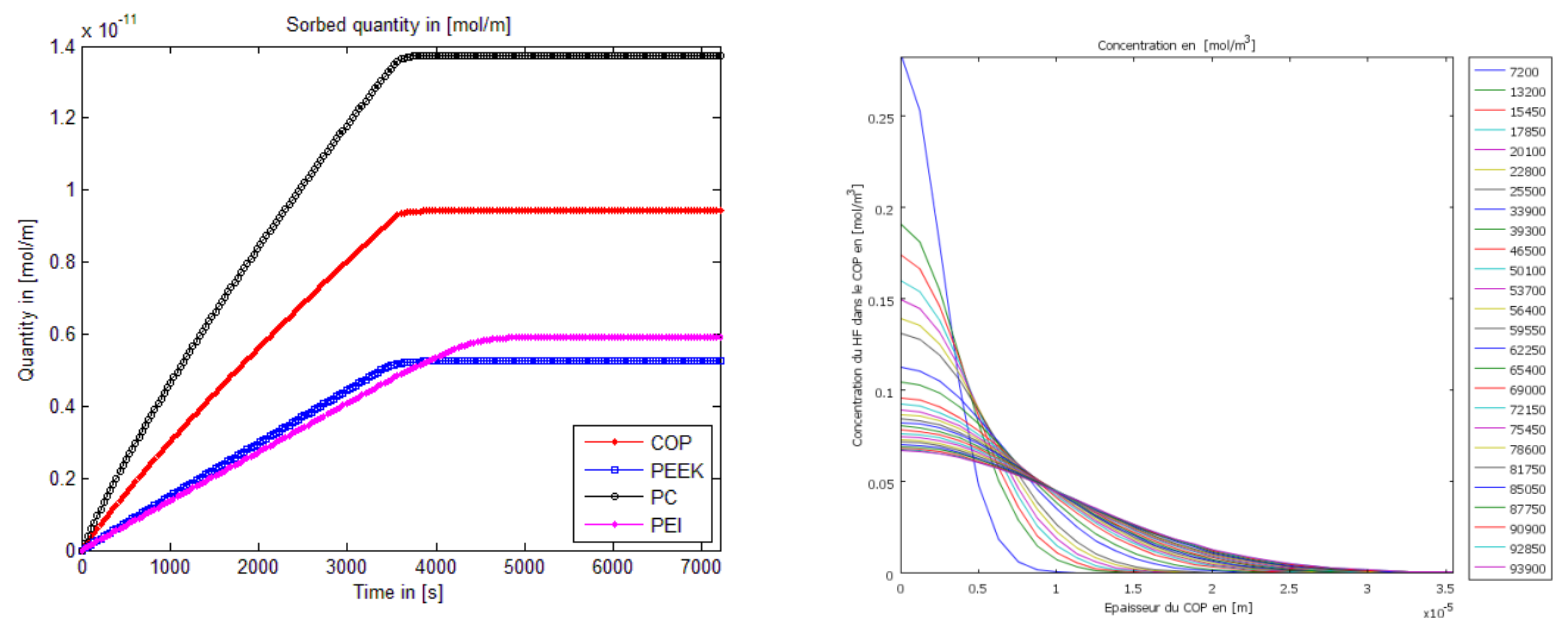

Fig. 5: Outgassing Process after Removing Wafer, Cleaning Effect after Purging FOUP with an Inert Gas. Outgassing Process after Removing Wafer, Cleaning Effect after Purging FOUP with an Inert Gas.
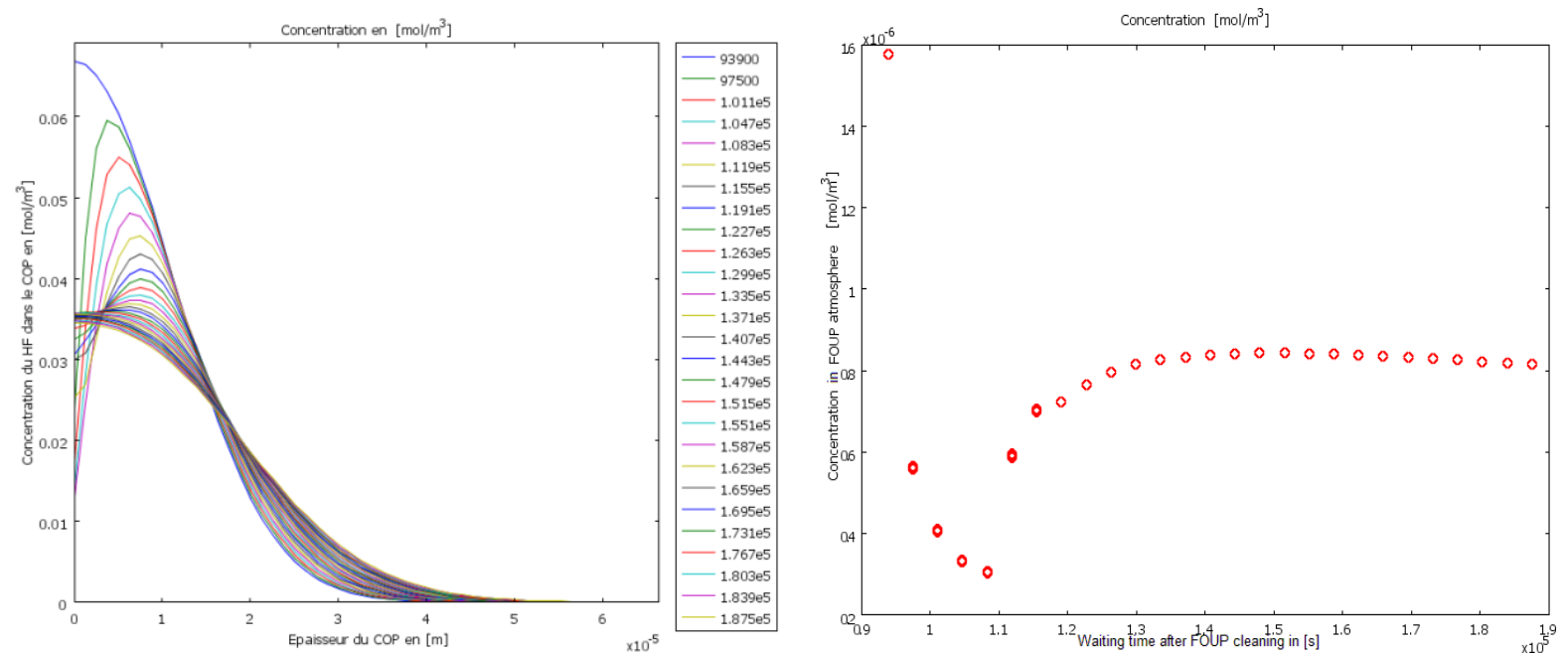

Fig. 6: Outgassing Process, Contamination after Cleaning Effect after Purging FOUP with an Inert Gas. Outgassing Process: Concentration in FOUP's Atmosphere after Cleaning by Using Cold Purging Method with an Inert Gas.

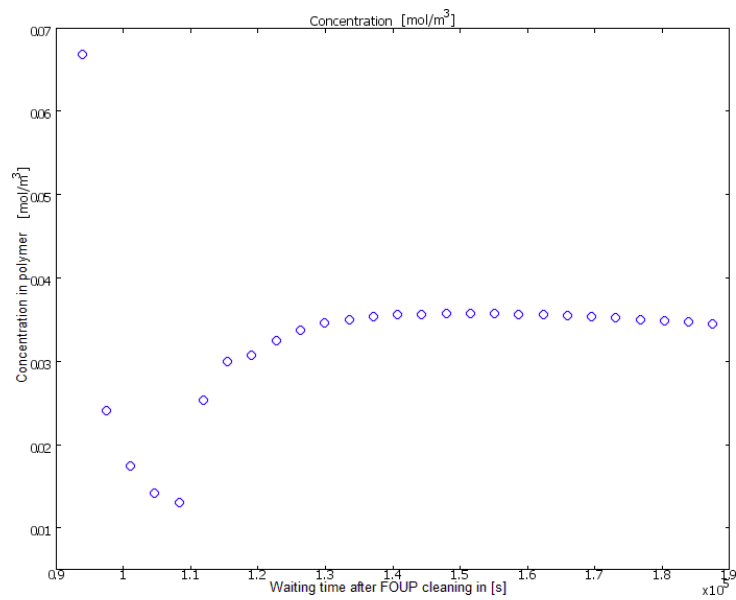

Fig. 7: Outgassing Process: Concentration in FOUP's Atmosphere after Cleaning by Using Cold Purging Method with an Inert Gas.

\section{Conclusions}

In The AMCs cross contamination model form a coupled partial differential equation? In this case, it is impossible to find explicit analytical solutions for the classical problems of mathematical physics. Most approaches are undertaken to the AMCs simulation a set of coupled partial differential equations has been solved by finite element method. The solution has been mainly determined by the applied boundary conditions as defined in each process. A considerable variety of boundary conditions has been implemented in Comsol multiphysics. Some basic mathematical properties 
have been analyzed which have to be fulfilled for self-consistent formulations of the boundary conditions in the device simulation. These formulations and implementations have been analyzed from the mathematical and the numerical points of view, illustrating both correct and inconsistent approaches with examples. An investigation of the use of the types of boundary conditions from both the mathematical and the numerical points of view are discussed. Consistency and convergence behavior has been illustrated with computational results and experimental measurement. The performance of the tools provides some following conclusions and remarks:

During the contamination processes the concentration is adsorb in the polymer and continue to diffuse during the outgassing process of the wafer and after the stopping outgas. This conditions is performed by using the Dirichlet to Neumann's boundary conditions. This first step conditioned all of the next step, it can be seen by simulation that we can obtained with this first approach a significant value of contamination level in the FOUP compared with the experimental measurement. The model is stable and consistent for these conditions but a mathematical development of these mixed boundary conditions is not yet developed in this work, but it will be performed with the next work. It was demonstrated that we have exactly the same solution if we separate the part of the Dirichlet to Neumann condition in two sub simulations i.e. we use just the Dirichlet condition and after that we apply the Neumann condition such that the initial condition is the last computed solution.

It can be seen that during the cleaning time the concentration of the contaminant near surface is outgassed and purged to the internal surface with the inert gas. We remarks that we have two part of the concentration gradient in the polymer, one part come to the internal surface (reversible contamination) and one part to volume (irreversible contamination). It can be seen that one part the contaminant continue to diffuse in the volume and one part of the contaminant come through to the surface absorbed by the purging system. Indeed the maximum of the concentration stay in the volume of the polymer. This residual part of the contamination can be moves (diffuses) in two direction: irreversible contamination in the volume of the polymer and the other parts ( the reversible parts) can constitutes the AMC source which contamined the new wafer in the FOUP atmosphere after cleaning.

It can be seen that the irreversible contamination results an accumulation with the residual concentration in the polymer. It can be proven that the levels of these parts increases and have effect in the cleaning time. The reversible part of the contamination accumulated in the polymer is the source of the contamination of the new wafer in the FOUP. With this process has been proved that plus the number of the cycle of the contamination increases the time of the cleaning time must increase too in order to take account of the residual contaminant adding during each cycle. We are measure to estimate the life time of the FOUP and the optimal time of the cleaning process in function of the cycle number.

The hot purging is benefit in term of efficiency of the contaminant removal during the cleaning process or decontamination of the FOUP. We have found through the simulation that the hot purging is more efficiency than the cold purging. The one problem is that this method induced long diffusion profile in the polymer i.e. the contaminant diffuse with maximum rate in the polymer because the phenomena is thermically activated. However, we need to determine for each contaminant the diffusion coefficient with temperature change. In fact, by using the approach based by the Arrhenius law, the value of the free enthalpy of each contaminant is needed. Another complex model not established in this work can be performed if the case we don't have the value of this free enthalpy.

It is expected that similar analysis can be carried out in other geometries taken account the FOUP scale and the wafers supports. Another approaches can be undertaken for example to take account the purging fluid flow circulation in the FOUP and to define for each contaminant a kinetic law at the interface. The simple domain considered here allows us to utilize a simply conditions and methods which simplifies the calculations and analysis and has given a best understanding of the AMCs cross contamination's dynamics. It is natural to speculate that when one considers other geometries, the use of some assumptions will be necessary.

\section{Acknowledgements}

We would like to acknowledge the contamination team at CEA/LETI/DTSI/SSURF-Grenoble-France who work on $300[\mathrm{~mm}] \mathrm{R} \& \mathrm{D}$ issues. N. Santatriniaina was financially supported by the Foundation of Rennes 1.

\section{References}

[1] Thi Quynh Nguyen, Hervé Fontaine, Yannick Borde, Véronique Jacob, Identification and quantification of FOUP molecular contaminants inducing defects in integrated circuits manufacturing, Microelectronic Engineering, vol. 105, 2013, pp. 124-129.

[2] Paola Gonzàlez-Aguirre, Hervé Fontaine, Carlos Beitia, Jim Ohlsen, Jorgen Lundgren, Poshin Lee, A comparative study of the HF sorption and outgas singability of different Entegris FOUP platforms and materials, Microelectronic Engineering, vol. 105, 2013, pp. 113-118.

[3] Hervé Fontaine, H. Feldis, A. Danel, S. Cetre, C. Ailhas, Impact of the volatile Acid Contaminant on Copper Interconnects, Electrical Performances. ECS Transactions, 25(5), 2009, pp. 78-86.

[4] Shih-Cheng Hu, Tzong-Ming Wu ,Hong-Chong Lin, Kwen Hsu, Design and evaluation of a nitrogen purge system for the front opening unifed pod (FOUP), Applied Thermal Engineering, vol. 27, pp. 1386-1393. 2007.

[5] Alemayeuhu Ambaw, Randolph Beaudry, Inge Bulens, Mulugeta Admasu Delele, Q. Tri Ho, Ann Schenk, Bart M. Nicolai, Pieter Verboven, Modeling the diffusion adsorption kinetics of 1-methylcyclopropene (1-MCP) in apple fruit and nontarget materials in storage rooms, Journal of Food Engineering, vol.102, 2011, pp. 257-265.

[6] Anli Geng, Kai-Chee Loh, Effects of adsoprtion kinetics and surface heterogeneity on band spreading in perfusion chromatography-a network model analysis, Chemical Engineering Science, vol. 59, 2004, pp. 2447-2465. 
[7] J. A. Boscoboinik, S.J. Manzi, V.D. Pereyra Adsorption-desorption kinetics of monomer-dimer mixture, Physics A, vol. 389, 2010, pp. 1317.1328 .

[8] H.Denny Kamaruddin, William J.Koros, Some observation about the application of Fick's first law for membrane separation of multicomponent mixtures, Journal of Membrane Science, vol. 135, 1997, pp. 147.159.

[9] Rico F. Tabor, Julian Eastoe, Peter J. Dowding, A two-step model for surfactant adsorption at solid surfaces, Journal of Colloid and Interface Science, vol. 346, 2010, pp. 424-428.

[10] Hiroki Nagaoka and Toyoko Imae, Ananlytical investigation of two-step adsorption kinetics on surfaces, Journal of Colloid and Interface Science vol. 264, 2003, pp. 335-342.

[11] Shengping Ding, William T. Petuskey, Solutions to Ficks second law of diffusion with a sinusoidal excitation, Solide State Ionics, vol. 109, 1998, pp. 101-110.

[12] K.J.Kuijlaars, C.R.Kleijin, H.E.A. van den Akker, Multi-component diffusion phenomena in multiple-wafer chemical vapor deposition reactors, The chemical Engineering Journal, vol. 57, 1995, pp. 127-136.

[13] Juergen Siepmann, Florence Siepmann, Modeling of diffusion controlled drug delivery,Journal of Controlled Release, vol. 161, 2012, pp. 351362 .

[14] J. Crank, The mathematics of diffusion, second edition, 1975 Clarendon Press, Oxford.

[15] Baptiste, Hiriart, Urruty, Optimisation et analyse convexe, Puf, 1998, page 11-12.

[16] R.Hirsch, C.C.Muller-Goymann, Fitting of diffusion coefficients in a three compartment sustained release drug formulation using a geneticalgorithm, International Journal of Pharmaceutics, vol. 120, 1995, pp. 229-234.

[17] Jacob Fish and Ted Belytschko, A first course of finite elements, north western university, USA, John Wiley and sons, Ltd, 2007.

[18] O.C. Zienkiewicz and R.L Taylor, The finite elements methods, volume 2, solid mechanics, fith edition, 2000.

\section{Appendix}

We introduce several notations which will be throughout this work. Let us:

$\nabla$. : Divergence operator

$\nabla$ : Gradient operator

$\mathrm{h}_{0}$ : Henry constant

$\mathrm{u}$ : Contaminant velocity

q: Source

$\mathrm{D}_{\mathrm{s}}$ : Diffusion coefficient in polymer at the temperature $\mathrm{T}$

$\mathrm{D}_{\mathrm{g}}$ : Diffusion coefficient in internal FOUPs atmosphere at the temperature $\mathrm{T}$

$\mathrm{D}_{\mathrm{s}}^{0}$ : Diffusion coefficient in polymer at the reference temperature

$\mathrm{D}_{\mathrm{g}}^{0}$ : Diffusion coefficient in internal FOUPs atmosphere at the reference temperature

$\mathrm{Q}^{\text {the }}$ : Experimental sorbed quantity of the contaminant in polymer

$\mathrm{Q}^{\mathrm{exp}}$ : Computed quantity of the contaminant in polymer

$\mathrm{C}^{\mathrm{s}}$ : Concentration in polymer

$\mathrm{C}^{\mathrm{g}}$ : Concentration in internal FOUPs atmosphere

$\mathrm{C}_{0}$ : Concentration on the wafer area

$\mathrm{n}$ : Outer unit normal vector

$\mathrm{N}_{0}^{\mathrm{g}}$ : Inlet concentration flux

$\mathrm{R}$ : Constant of perfect gas

$\mathrm{T}:$ Temperature

$\mathrm{E}_{0}$ : Free enthalpy of the contaminant

$\mathrm{F}_{\mathrm{a}}\left(\mathrm{C}_{0} ; \mathrm{C}^{\mathrm{g}}\right)$ : Cold boundary condition

$\mathrm{F}_{\mathrm{a}}^{\mathrm{T}}\left(\mathrm{C}_{0} ; \mathrm{C}^{\mathrm{g}}\right)$ : Boundary condition with heat effect

$\mathrm{H}(\mathrm{t}-\varepsilon)$ : Heaviside function with delay $\varepsilon$

$\Omega_{\mathrm{s}}:$ Polymer domain

$\Omega_{\mathrm{g}}$ : Contaminant domain

AMCs: Airborne Cross contamination

FOUP: Front Opening Unified Pod

B.C: Boundary Condition

I.C: Initial Condition

AFM: Atomic Force Microscopy

REV: Representative Elementary Volume 